CÂNDIDO, V. A.; PINTO, L. V. A.; BOGARIMP, P. de C.; ROSAS, D. da; SILVA, R. M.; BARBOSA, J. M. N. Sistema agroflorestal para recomposição de reserva legal em propriedades de agricultores familiares. Revista Agrogeoambiental, Pouso Alegre, v. 8, n. 2, p. 65-72, Jun. 2016. DOI: http://dx.doi.org/10.18406/2316-1817v8n22016821

\title{
- Sistema agroflorestal para recomposição de reserva legal em propriedades de agricultores familiares
}

\author{
Vinícius Alves Cândido ${ }^{1}$ \\ Lilian Vilela Andrade Pinto ${ }^{2}$ \\ Paula de Castro Bogarim³ \\ Silas Dias da Rosa ${ }^{4}$ \\ Rafael Monti Silva 5 \\ Joice Magali Nunes Barbosa ${ }^{6}$
}

\section{Resumo}

Os sistemas agroflorestais são preconizados como uma solução alternativa para a recuperação de áreas degradadas. Assim, o presente estudo teve como objetivo implantar e monitorar um protótipo de sistema agroflorestal (SAF) modelo silviagrícola, avaliando o desenvolvimento inicial de três espécies florestais nativas, comparando-o ao modelo de plantio convencional. O modelo estatístico adotado foi o fatorial 2 (tratamentos: plantio convencional de espécies florestais e plantio em sistema agroflorestal) x 3 (espécies: Lonchocarpus muehlbergianus Hassl. - embira-de-sapo, Cytharexyllum myrianthum Cham. - tucaneira, Schinus terebinthifolius Raddi - aroeira-pimenteira) com 3 repetições, seguindo o delineamento inteiramente casualizado. A espécie que obteve maior crescimento, tanto no sistema convencional quanto no silviagrícola, foi a C. myrianthum Cham. A espécie L. mueh/bergianus Hassl. apresentou o menor crescimento em ambos os tratamentos. A espécie S. terebinthifolius Raddi atingiu maior crescimento no sistema convencional. Foram colhidos 2,17 t ha-1 de feijão e 1 t ha-1 de banana. Como não foi observada diferença significativa no crescimento da altura e do diâmetro à altura do solo (DAS) das espécies entre o plantio convencional e silviagrícola, o SAF mostra-se como alternativa para a recomposição de reserva legal no sul de Minas Gerais, possibilitando maior aproveitamento da área a ser recuperada, sendo o uso das entrelinhas para produzir culturas anuais uma alternativa viável.

Palavras-chave: Agroecologia. Recursos ambientais. Área degradada.

\section{Introdução}

A região sul de Minas Gerais possui em grande parte de seus municípios uma matriz produtiva rural, sendo a agropecuária uma das principais atividades econômicas da região (PEDINI, 2005; SILVEIRA et al., 2006).

1 Universidade Federal de Lavras, mestrando em Engenharia Florestal. Lavras, MG, Brasil. viniciuseco@posgrad.com.br. Departamento de Ciências Florestais. Caixa Postal 3037, Lavras, MG, CEP 37200-000.

2 IFSULDEMINAS - Campus Inconfidentes, professora pesquisadora do Centro de Procedimentos Ambientais. Inconfidentes, Minas Gerais, Brasil. Iilian.vilela@ifsuldeminas.edu.br. Praça Tiradentes, 416, Centro, Inconfidentes, MG, CEP 37576-000.

3 Universidade Federal de Lavras, pós-graduanda em Educação Ambiental e Espaços Educadores Sustentáveis. Lavras, MG, Brasil. bogarim.ifet@gmail.com. Rua Juiz Dr José Ferreira da Paixão, 12, Centro, Três Corações, MG, CEP 37410-000.

4 IFSULDEMINAS - Campus Inconfidentes, tecnólogo em Gestão Ambiental. Inconfidentes, MG, Brasil. silasdias_sdro@hotmail.com. Rua Prof. Arthur Luponi, 326, Nossa Senhora Aparecida, Cambuí, MG, CEP 37600-000.

5 IFSULDEMINAS - Campus Inconfidentes, tecnólogo em Gestão Ambiental. Inconfidentes, MG, Brasil. rafaelmontisilva@yahoo.com.br. Rua Paiva Junior, 45, Centro, Pedralva, MG, CEP 37520-000.

6 IFSULDEMINAS - Campus Inconfidentes, tecnóloga em Gestão Ambiental. Inconfidentes, MG, Brasil. joice.mnb@gmail.com. Rua Prof. Arthur Luponi, 326, Nossa Senhora Aparecida, Cambuí, MG, CEP 37600-000. 
A gestão incorreta dos recursos naturais provoca alterações na flora, solo, água, fauna e clima, podendo causar perturbação e degradação ambiental que são capazes de interferir na produção e manutenção da qualidade de vida dos moradores das bacias hidrográficas modificadas (GOULART; CALLISTO, 2003).

Diversas são as consequências advindas das alterações da paisagem, tanto no meio urbano quanto no rural, e para combater as consequências relacionadas à ausência de vegetação nativa e minimizar os impactos sobre o meio ambiente é necessário reformular a aplicação dos instrumentos das políticas públicas ambientais (RAMOS FILHO; FRANCISCO, 2004).

Ações exercidas por órgãos ambientais, responsáveis pela recuperação, conservação e preservação dos recursos ambientais, são orientadas pelo modelo fiscalizador, no qual a multa ambiental é o instrumento utilizado na tentativa de estabelecer novas áreas de reserva legal (GORSKI, 2009).

As condições de incentivo aos produtores rurais, proprietários de pequenas propriedades, para aderirem às práticas de recuperação ambiental pressupõem novas estratégias. Medidas que estabeleçam o desenvolvimento de programas para subsidiar o gerenciamento ambiental rural devem ser adotadas (MATTOS et al., 2007). Para atingir esses objetivos, é necessário reformular a aplicação dos instrumentos das políticas públicas ambientais (BITTENCOURT, 2002).

São necessários, portanto, investimentos em estudos e divulgação dos sistemas agroflorestais, oferecendo uma alternativa viável aos agricultores familiares para recuperação de áreas degradadas no sul de Minas Gerais.

Não basta apenas fiscalizar, é preciso fornecer aos agricultores familiares subsídios, redução de impostos e outras formas de compensação por serviços ambientais prestados (SANTANA et al., 2009).

O objetivo deste estudo foi avaliar o potencial de três espécies nativas da Mata Atlântica, plantadas em sistema agroflorestal modelo silviagrícola, para a recuperação de áreas degradadas, em especial, para a recomposição de reserva legal.

\section{Material e métodos}

O experimento foi conduzido na Fazenda Escola do Instituto Federal do Sul de Minas Gerais Campus Inconfidentes/MG. A área de estudo está localizada a uma altitude de $879 \mathrm{~m}$, tem posição marcada pelas coordenadas geográficas de 22018'37" latitude sul e 46¹9'47" longitude oeste. 0 clima da região, segundo classificação de Koëppen, é o Cwb, clima mesotérmico caracterizado por verões brandos e úmidos. A precipitação média anual varia de 1.400 a $1.800 \mathrm{~mm}$, o período seco tem duração de 2 a 3 meses e coincide com os meses mais frios, nos quais a temperatura média é inferior a $18^{\circ} \mathrm{C}$.

Antes da implantação do experimento, realizou-se roçagem e capina na área para reduzir a presença de plantas espontâneas (Brachiaria decumbens Staff - braquiária, Ciperus rotundus Linnaeus - tiririca, Cynodon dactylon L. - grama-seda). Troncos de árvores e resíduos orgânicos de origem animal também foram encontrados na área e retirados.

Foram abertos berços de 40 × 40 × $40 \mathrm{~cm}$ para plantio das mudas florestais (L. mueh/bergianus Hassl. - embira-de-sapo, C. myrianthum Cham. - tucaneira, S. terebinthifolius Raddi - aroeira-pimenteira) e berços de $80 \times 80 \times 80 \mathrm{~cm}$ para plantio das mudas frutíferas (Psidium guajava L. - goiaba, Musa paradisíaca L.- banana), seguindo o espaçamento $3 \times 2 \mathrm{~m}$. Foram utilizados $2 \mathrm{~kg}$ de esterco bovino curtido por cova.

0 modelo estatístico adotado foi o fatorial 2 (tratamentos: plantio convencional de espécies florestais e plantio em sistema agroflorestal) x 3 (espécies nativas arbóreas: L. muehlbergianus Hassl., 
C. myrianthum Cham., S. terebinthifolius Raddi), com 3 repetições por espécie, passando por análise do teste $T(P>0,05)$, seguindo o delineamento inteiramente casualizado (DIC). As médias da altura, do diâmetro à altura do solo e da área de copa das espécies arbóreas foram comparadas pelo teste de médias de Scott-Knott.

Ressalta-se que durante a condução do experimento houve mortalidade de alguns indivíduos arbóreos, sendo necessário realizar o replantio. No período de maio a junho de 2011 , todos os indivíduos da espécie Cabralea canjerana (Vell.) Mart. (canjerana) morreram, o que resultou na substituição da espécie, em agosto de 2011, pelo plantio de mudas da espécie S. terebinthifolius Raddi (aroeira-pimenteira).

O modelo agroflorestal adotado foi o silviagrícola, definido por Macedo (2000) como a combinação de árvores, arbustos ou palmeiras com espécies agrícolas. Para a composição desse sistema, foram introduzidas nas linhas, no mês de agosto de 2011, as espécies frutíferas Bactris gasipaes (pupunha), Eriobothrya japonica (Thunb.) Lind (ameixa), Prunus myrtifolia (L.) Urb (pessegueiro-bravo), Eugenia pyriformis Cambess (uvaia); nas entrelinhas, durante o mês de setembro de 2011, em uma área de 0,012 ha, foi inserido 1 (um) kg de Phaseolus vulgaris L. (feijão-carioquinha) variedade canário e 10 unidades de manivas de Manihot utilíssima Pohl (mandioca).

Para melhor conhecimento sobre a concentração de nutrientes no solo, foram coletadas amostras de acordo com a metodologia da EMBRAPA (2010), não tendo sido realizada correção e adubação do solo, visto que anteriormente à implantação do experimento, a área foi utilizada para descarte de fezes de aves e restos de podas (galhos, folhas, troncos), tornando o solo rico em nutrientes e matéria orgânica. Durante 16 meses, foram realizadas mensurações da altura e diâmetro à altura do solo das espécies florestais, a partir de aferições realizadas com fita métrica e paquímetro, respectivamente, a cada 30 dias. A mensuração da altura consistiu na medida da altura do chão ao ápice das mudas. Já a mensuração do diâmetro à altura do solo (DAS) consistiu da medida do diâmetro rente ao solo. Esses indicadores são frequentemente utilizados em pesquisas que avaliam o desenvolvimento comparativo entre espécies em áreas em recuperação, podendo destacar os trabalhos de Resende et al. (2015), Sampaio et al. (2012), Leles et al. (2011) e Moraes et al. (2006).

As atividades de manejo do experimento (capina, coroamento e controle de formigas) foram realizadas quando necessário. Para o controle de formigas, utilizou-se de bioinseticida produzido à base de borra de café, na proporção 2 litros d'água para 500 gramas de borra de café.

\section{Resultados e discussão}

Entre as espécies estudadas, a que obteve maior crescimento tanto no sistema convencional quanto no silviagrícola foi a $C$. myrianthum Cham. (tucaneira), atingindo 4,75 m (sd =0,82 $\mathrm{m}$ ) de altura (Figura 1A) e $125 \mathrm{~mm}(\mathrm{sd}=42 \mathrm{~mm}$ ) de diâmetro à altura do solo (Figura 1B) aos 16 meses, não havendo diferença estatística entre os sistemas de plantio $(P>0,05)$. 0 valor da altura alcançado pela espécie pode ser considerado excelente por se encontrar próximo ao valor de $5 \mathrm{~m}$ de altura observado por Padovan et al. (2009) aos 3 anos de idade em um sistema agroflorestal na região sul de Mato Grosso do Sul. Por ser uma espécie pioneira, conforme classificação de Sampaio et al. (2012) e Leles et al. (2011), era de se esperar um bom desenvolvimento da espécie nos atributos avaliados (altura e diâmetro à altura do solo) nos dois modelos de plantio (convencional e silviagrícola) pelo fato de as condições ambientais (temperatura, precipitação, altitude, tipo de solo, fertilidade e grau de compactação) no solo do local de instalação das parcelas experimentais serem iguais, 
devido à reduzida distância entre as parcelas e aos tratos culturais (adubação, controle de formiga, coroamento e podas das espécies florestais) terem sido padronizados.

O diferencial entre os sistemas de plantio foi o arranjo espacial em que o modelo convencional concentra menor número de indivíduos em relação ao sistema agroflorestal, sendo que essa combinação de espécies florestais, frutíferas e culturais anuais não provocou diferença no crescimento das espécies florestais nativas, sendo um excelente indicador para o uso do SAF modelo silviagrícola para a recuperação de áreas degradadas, podendo unir o conceito de preservação da biodiversidade com o de produção sustentável.

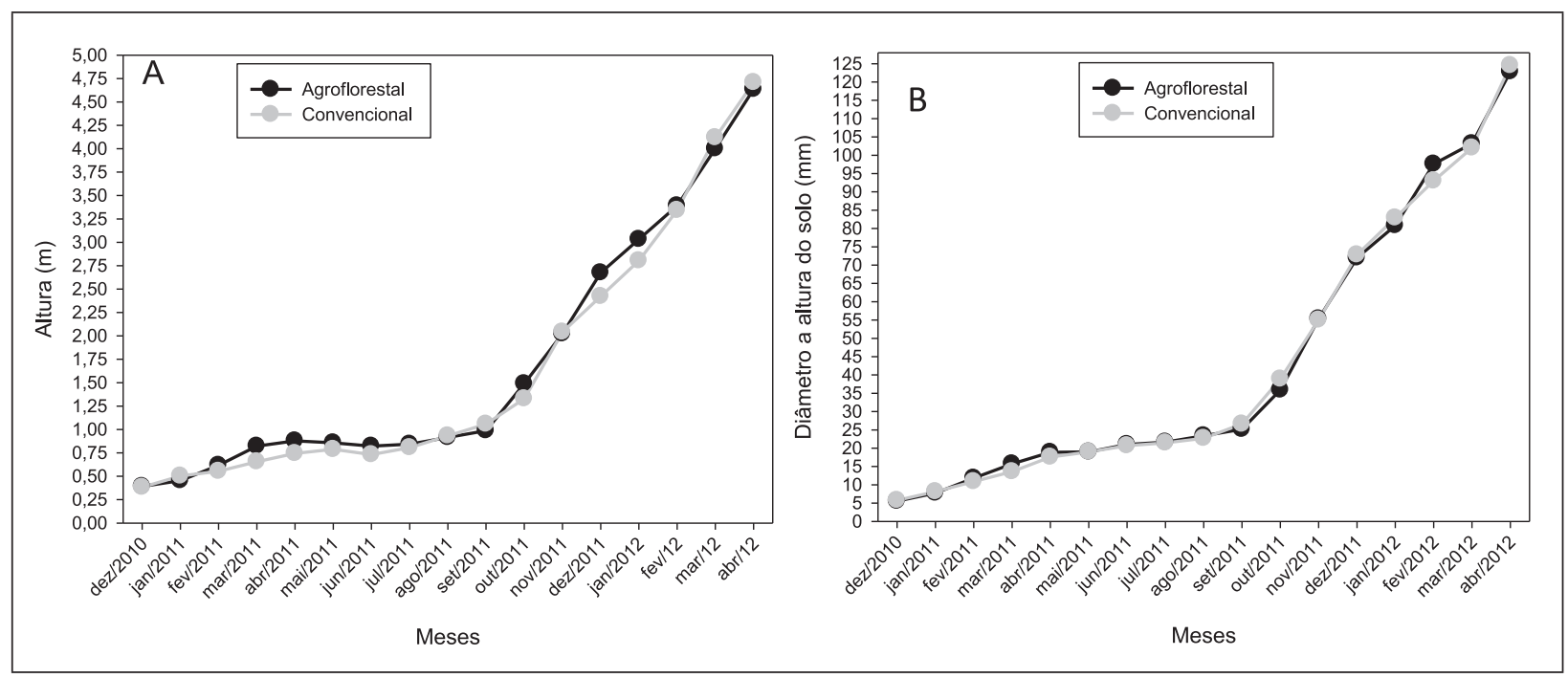

Figura 1. Desenvolvimento de C. myrianthum Cham.: A) Altura; B) Diâmetro à altura do solo Fonte: Elaboração dos autores

A espécie S. terebinthifolius Raddi (pimenteira) obteve maior crescimento no sistema convencional, atingindo 2,75 $\mathrm{m}(\mathrm{sd}=0,46 \mathrm{~m}$ ) de altura e $32 \mathrm{~mm}(\mathrm{sd}=5,52 \mathrm{~mm}$ ) de diâmetro aos 16 meses (Figuras 2A e 2B). No sistema silviagrícola, a espécie alcançou 2,35 m ( $\mathrm{sd}=0,82 \mathrm{~m}$ ) de altura e $35 \mathrm{~mm}$ (sd = $79 \mathrm{~mm}$ ) de diâmetro (Figuras 2A e 2B).

Mesmo tendo ocorrido diferença estatística $(P>0,05)$ no desenvolvimento da espécie em altura, a partir de fevereiro de 2012, entre os sistemas de plantio, pode-se considerar que a espécie apresentou bom desenvolvimento em ambos os sistemas (convencional e silviagrícola), em razão do seu crescimento ser muito variado em diferentes experimentos. Como exemplo, destacam-se os resultados de Souza et al. (2004) que detectaram, aos 18 meses, 3,57 m e de Carvalho (2003) que observou 0,99 m de altura aos 5 anos de idade. Para Souza (2004), grandes amplitudes no crescimento, muitas vezes, são comuns para espécies nativas desprovidas de melhoramento genético. Devido ao seu potencial adaptativo, a espécie S. terebinthifolius Raddi se desenvolve em diferentes condições ambientais, de solos encharcados a terrenos arenosos e secos (Souza, 2013). Estudando o desenvolvimento da espécie em ambiente de mata ciliar na região do rio São Francisco, Pedroso et al. (2004) e Ferreira et al. (2009) encontraram, aos 12 meses, indivíduos com $38 \mathrm{~cm}$, sendo possível afirmar que essas diferenças no crescimento fazem parte do comportamento normal da espécie. 


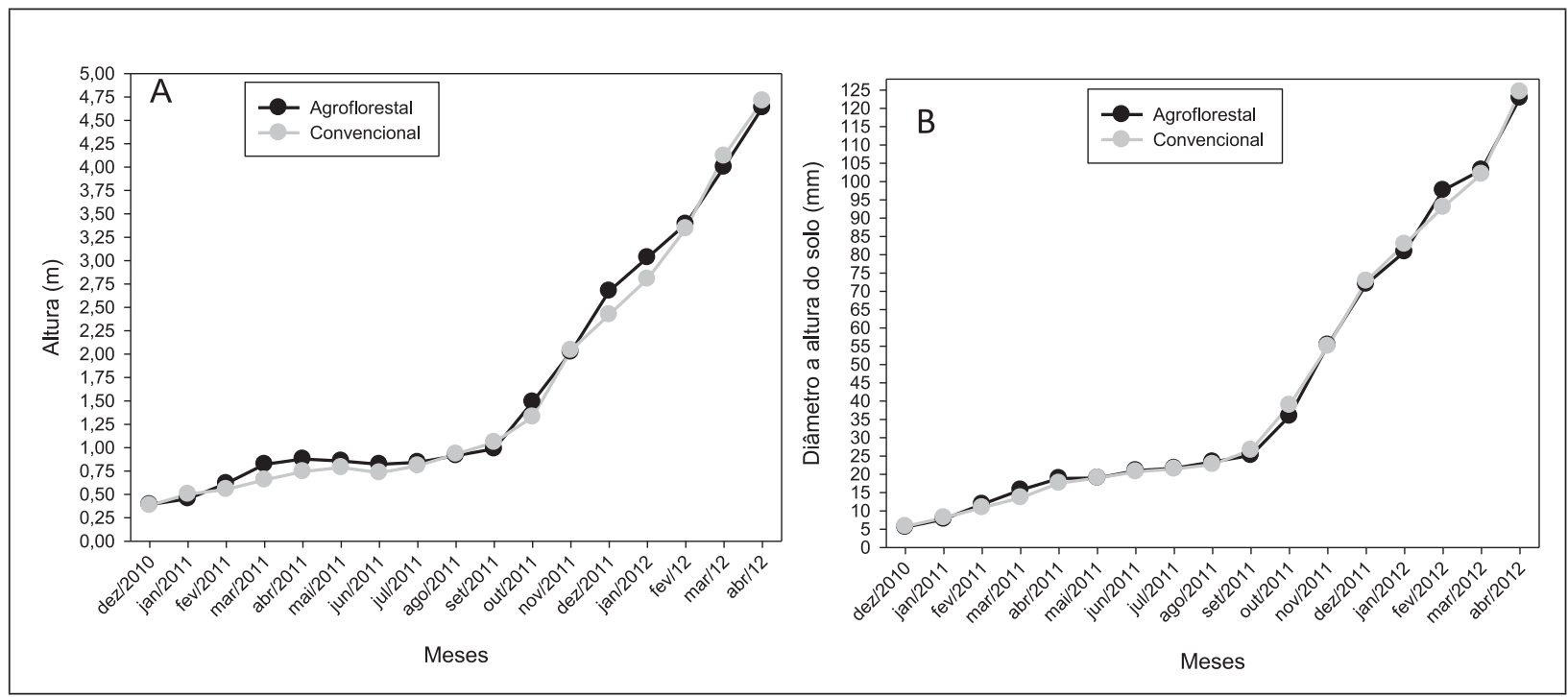

Figura 2. Desenvolvimento de S. terebinthifolius Raddi: A) Altura; B) Diâmetro a altura do solo Fonte: Elaboração dos autores

A espécie L. mueh/bergianus Hassl. (embira-de-sapo) obteve o menor crescimento entre as espécies, atingindo altura mínima de 1,90 $\mathrm{m}(\mathrm{sd}=0,52 \mathrm{~m}$ ) e $32 \mathrm{~mm}$ no sistema silviagrícola aos 16 meses, não diferindo estatisticamente $(P>0,05)$ do observado no sistema convencional (Figuras $3 \mathrm{~A}$ e $3 \mathrm{~B}$ ). Mesmo tendo sido o menor valor de altura observado entre as espécies estudadas, 0 crescimento em altura alcançado pela espécie pode ser considerado bom e dentro da normalidade, visto Oliveira (2011) ter observado altura de 1,00 m aos 6 meses em regiões de baixada. Para Torres (2008), por ser uma espécie climáxica, L. muehlbergianus Hassl. possui baixa tolerância ao sol e crescimento lento, por isso, seu baixo crescimento inicial pode ser considerado normal.

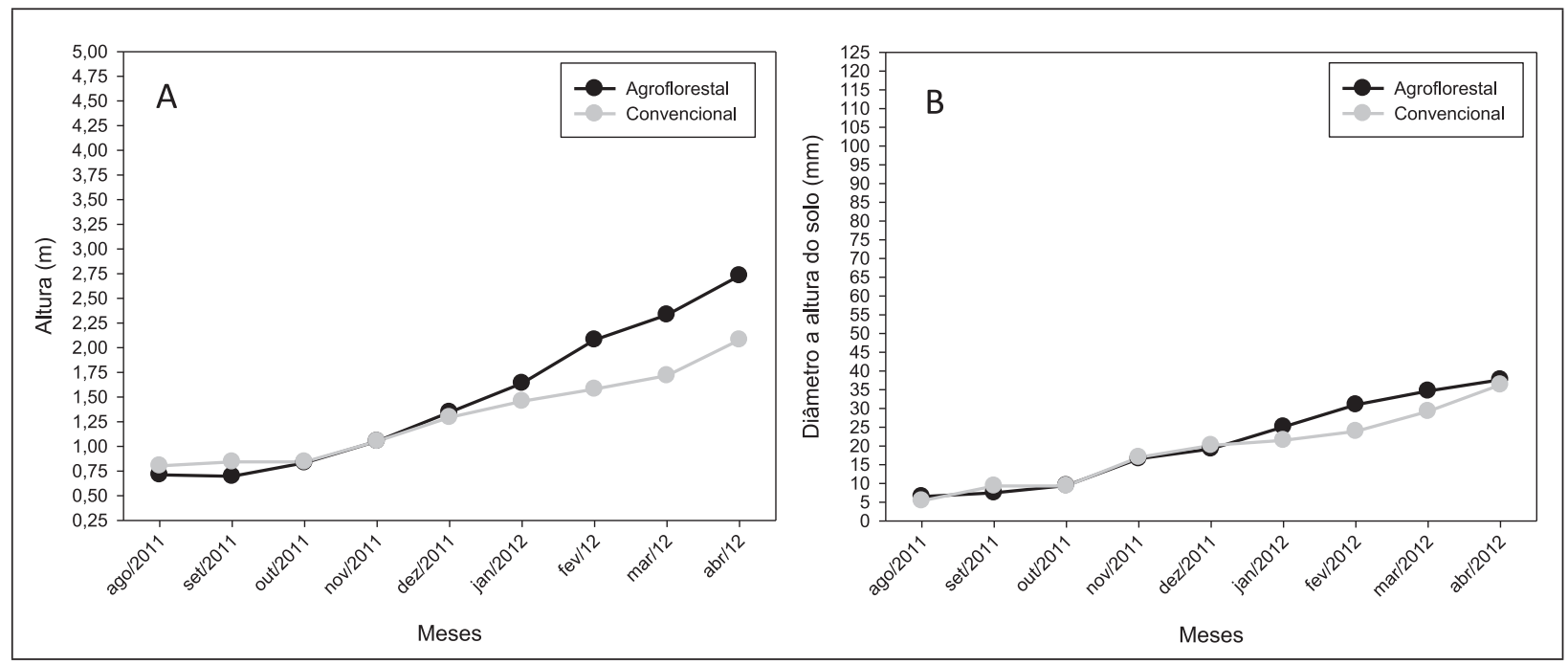

Figura 3. Desenvolvimento de L. muehlbergianus Hassl.: A) Altura; B) Diâmetro à altura do solo Fonte: Elaboração dos autores

Além do desenvolvimento das espécies arbóreas no sistema silviagrícola, ainda foram colhidos $26 \mathrm{~kg}$ de feijão e $12 \mathrm{~kg}$ de banana em uma área de 0,012 ha, o equivalente a 2,17 t ha-1 e $1 \mathrm{t} \mathrm{ha}^{-1}$, respectivamente. A produtividade média brasileira de feijão é de 93 t. ha-1 em lavouras da agricultura familiar. Para a cultura da banana, a produtividade média é de 14,298 t. ha-1 (IBGE, 2010). 
O cultivo de culturas agrícolas e frutíferas em associação a espécies nativas oferece aos agricultores familiares a possibilidade de diversificação da produção, colhendo alimentos variados em diferentes épocas do ano, agregando renda por meio da comercialização da colheita excedente, com produtividade equiparada ao plantio convencional.

De acordo com Da Croce (1992), o cultivo de feijão nas entrelinhas contribui para cobertura do solo e proporciona rentabilidade econômica. Dessa forma, o sistema agroflorestal apresentou-se como um grande potencial para produção de alimentos, podendo produzir $P$. vulgaris (feijão carioquinha), M. sp (banana), M. utilísima Krantz (mandioca) e outros.

O sistema agroflorestal possibilita a produção de alimentos associada à recuperação de áreas degradadas, incentiva a produção diversificada e o consumo de alimentos saudáveis, tornando possível a venda do excedente, quebrando o paradigma sobre a improdutividade das pequenas propriedades que realizam a recomposição das áreas de reserva legal.

Para que as atividades agropecuárias sejam desenvolvidas com longevidade, conservando e preservando os recursos naturais e o meio ambiente, torna-se necessário firmar parcerias com Instituições de Ensino, investir em assistência técnica e extensão rural inovadora e promover, por meio da agroecologia, a transformação na maneira de pensar e na forma agir.

\section{Conclusão}

O sistema agroflorestal modelo silviagrícola mostra-se como alternativa para a recomposição de reserva legal no sul de Minas Gerais, possibilitando maior aproveitamento da área a ser recuperada, sendo o uso das entrelinhas para produzir culturas anuais uma alternativa viável.

\section{Agradecimentos}

À FAPEMIG pela bolsa de iniciação científica (Edital 12/2011) e aos membros do projeto APQ -01455-14 pelo apoio.

\section{Agroforestry system for recovering legal reserve in family farm properties}

\section{Abstract}

Agroforestry systems are recommended as an alternative to recover degraded areas. Thus, this study aimed to implement and monitor an agroforestry system prototype (SAF in Portuguese), agroforestry model, evaluating the initial development of three native species, comparing this system to the planting conventional model. The adopted statistical model was the factorial 2 (treatments: planting forest species by the conventional system and planting them by the agroforestry system) x 3 (species: Lonchocarpus muehlbergianus Hassl., Cytharexyllum myrianthum Cham., Schinus terebinthifolius Raddi), 3 repetitions, following a completely randomized design. The species that reached the highest growth in both the conventional and agroforestry system was the $C$. myrianthum Cham.. The L. muehlbergianus Hassl. species had the lowest growth in both treatments. The S. terebinthifolius Raddi species showed a higher growth in the conventional system. 2,17 $\mathrm{t} \mathrm{ha}^{-1}$ of beans and $1 \mathrm{t} \mathrm{ha}^{-1}$ of bananas were harvested. As there was neither significant difference at the height growth of the species nor at the diameter at ground level (DGL) on both conventional and agroforestry 
systems, the SAF is presented as an alternative for rebuilding reserves in southern Minas Gerais as it allows greater use of the area to be recovered, being growing crops in inter-row one example.

Keywords: Agroecology. Environmental resources. Degraded area.

\section{Referências}

BITTENCOURT, G. Agricultura familiar e agronegócio. In: LIMA, D. M. A.; WILKINSON, J. Inovação nas tradições da agricultura familiar. Brasília: CNPQ, p.85-94, 2002.

CARVALHO, P. E. R. Espécies Arbóreas Brasileiras. Brasília: Embrapa Informação Tecnológica; Colombo: Embrapa Florestas. 2003. 739 p.

DA CROCE, D. M.; NADAL, R. de. Viabilidade técnico econômica de sistemas de produção de erva-mate consorciada com culturas anuais. In: ENCONTRO BRASILEIRO DE ECONOMIA E PLANEJAMENTO FLORESTAL, Curitiba. Anais... Colombo: EMBRAPA, 1992.

EMBRAPA - Empresa Brasileira de Pesquisa Agropecuária. Método para coleta de amostras de solos para análise. [s.I.:s.n.] 2008. Disponível em: <http://www.cnps.embrapa.br/servicos/metodo_coleta.html>. Acesso em 23 set. 2010.

FERREIRA, R. A.; SANTOS, P. L.; ARAGÃO, A. G. de; SANTOS, T. I. S.; SANTOS NETO, L. M.; REZENDE, A. M. S. Semeadura direta com espécies florestais na implantação de mata ciliar no Baixo São Francisco em Sergipe. Scientia Forestalis, Piracicaba, v. 37, n. 81, p. 37-46, mar. 2009.

GORSKI, J. A. Tributação ambiental: desafios para uma política de proteção ao meio ambiente. 2009. 87p. Dissertação (Mestrado em Desenvolvimento e Meio Ambiente). Universidade Federal da Paraíba, João Pessoa, 2009.

GOULART, M. E.; CALLISTO, M. 2003. Bioindicadores de qualidade de água como ferramenta em estudos de impacto ambiental. Revista da FAPAM, ano 2, n. 1.

IBGE. Ministério do Planejamento, Orçamento e Gestão. Produção agrícola municipal culturas temporárias e permanentes 2010. Rio de Janeiro. 2010.

LELES, P. S. S.; ABAURRE, G. W.; ALONSO, J. M.; NASCIMENTO, D. F.; LISBOA, A. C. Crescimento de espécies arbóreas sob diferentes espaçamentos em plantio de recomposição florestal. Scientia Forestalis (IPEF), v. 39, p. 231-239, 2011.

MACEDO R. L. G. Princípios básicos para o manejo sustentável de sistemas agroflorestais. Universidade Federal de Lavras, Lavras, MG, 2000.

MATTOS, A. D. M.; JACOVINE, L. A. G.; VALVERDE, S. R.; SOUZA, A. L.; SILVA, M. L.; LIMA, J. E. Valoração ambiental de áreas de preservação permanente da microbacia do ribeirão São Bartolomeu no município de Viçosa, MG. Revista Árvore, v. 31, n. 2, p. 347-353, 2007.

MORAES, L. F. D.; ASSUMPÇÃO, J. M. ; LUCHIARI, C. ; PEREIRA, T. S. Plantio de espécies arbóreas nativas para a restauração ecológica na Reserva Biológica de Poço das Antas, Rio de Janeiro, Brasil. Rodriguesia, v. 57, p. 477-489, 2006. 
OLIVEIRA, N. M. Estabelecimento de espécies florestais a partir do plantio em diferentes posições do relevo. Universidade Federal Rural do Rio de Janeiro. Instituto de Florestas. Seropédica, RJ. 2011.

PADOVAN, M. P.; PEREIRA, Z. V.; FERNANDES, S. S. L.; SALOMÃO, G. B.; LOBTCHENKO, G.; SILVA, S. M. de. Performance e fitossociologia de espécies arbóreas em um sistema agroflorestal sob bases ecológicas na região sul de Mato Grosso do Sul. Embrapa Agropecuária Oeste, Dourados, MS. 2009.

PEDINI, S. Certificação e comercialização de cafés da agricultura familiar. Informe Agropecuário, Belo Horizonte, v. 26, p. 118-124, 2005. Edição especial.

PEDROSO, K. B.; ANGELO, A. C.; KASSEBOHEMER, A.L.; GROSSI, F.; SOUZA, L. P. de. A importância do elemento arbóreo em SAFs: desenvolvimento de seis espécies em solos semi-hidromórficos. In: CONGRESSO BRASILEIRO DE SISTEMAS AGROFLORESTAIS, 5., 2004, Curitiba. Anais... Colombo: Embrapa Florestas, p. 455-457, 2004.

RAMOS FILHO, L. O.; FRANCISCO, C. E. S. Legislação florestal, sistemas agroflorestais e assentamentos rurais em São Paulo: Restrições ou oportunidades. In: CONGRESSO BRASILEIRO DE SISTEMAS AGROFLORESTAIS, 5., 2005, Curitiba. Anais... Colombo: Embrapa, p. 211-213, 2004.

RESENDE, L. A. ; PINTO, L. V. A. ; SANTOS, E. C. Crescimento e sobrevivência de espécies arbóreas em diferentes modelos de plantio na recuperação de área degradada por disposição de resíduos sólidos urbanos. Revista Árvore (Online), v. 39, p. 147-157, 2015.

SAMPAIO, M. T. F. ; POLO, M. ; BARBOSA, W. Estudo do crescimento de espécies de árvores semidecíduas em uma área ciliar revegetada. Revista Árvore (Online), v. 36, p. 879-885, 2012.

SANTANA, A. C.; FERREIRA, P. A.; ALENCAR, D. Diversificação da agricultura familiar no sul de Minas Gerais: uma análise da percepção de professores e pesquisadores. $47^{\circ}$ CONGRESSO DA SOCIEDADE BRASILEIRA DE ECONOMIA, ADMINISTRAÇÃO E SOCIOLOGIA RURAL. Anais... Porto Alegre, 2009.

SILVEIRA, M. A.; CARON, D.; MARQUES, P. E. M.; IAMAMOTO, A. T. V. Análise da multifuncionalidade e desenvolvimento territorial em áreas de cafeicultura familiar no sul de Minas Gerais. Estudo de Caso, Embrapa Meio Ambiente, Jaguariúna, p 1-16. 2006.

SOUZA, M. N. Degradação e recuperação ambiental e desenvolvimento sustentável. 2004. Dissertação (Mestrado em Ciência Florestal). Universidade Federal de Viçosa Viçosa, MG, 2004.

TORRES, J. A. P.; SCHIAVINATO, M. A. Crescimento, eficiência fotossintética e eficiência do uso da água em quatro espécies de leguminosas arbóreas tropicais. 2008. Hoehnea. p. 395-404.

\section{Histórico editorial}

Submetido em: 24/03/2015

Aceito em: 16/06/2015 